\title{
Consumption of Wild Rice (Zizania latifolia) Prevents Metabolic Associated Fatty Liver Disease through the Modulation of the Gut Microbiota in Mice Model
}

\author{
Xiao-Dong Hou ${ }^{1}$, Ning Yan ${ }^{1}$, Yong-Mei Du ${ }^{1}$, Hui Liang ${ }^{2}$, Zhong-Feng Zhang ${ }^{1}$ and \\ Xiao-Long Yuan ${ }^{1, *}$ \\ 1 Tobacco Research Institute of Chinese Academy of Agricultural Sciences, Qingdao 266109, China; \\ houxiaodong@caas.cn (X.-D.H.); yanning0532@163.com (N.Y.); duyongmei0532@163.com (Y.-M.D.); \\ zhangzhongfeng@caas.cn (Z.-F.Z.) \\ 2 College of Public Health, Qingdao University, Qingdao 266101, China; qdlianghui@qdu.edu.cn \\ * Correspondence: rayrock@126.com; Tel.: +86-(532)-66715079
}

Received: 29 June 2020; Accepted: 23 July 2020; Published: 29 July 2020

\begin{abstract}
Metabolic associated fatty liver disease (MAFLD) due to excess weight and obesity threatens public health worldwide. Gut microbiota dysbiosis contributes to obesity and related diseases. The cholesterol-lowering, anti-inflammatory, and antioxidant effects of wild rice have been reported in several studies; however, whether it has beneficial effects on the gut microbiota is unknown. Here, we show that wild rice reduces body weight, liver steatosis, and low-grade inflammation, and improves insulin resistance in high-fat diet (HFD)-fed mice. High-throughput $16 \mathrm{~S}$ rRNA pyrosequencing demonstrated that wild rice treatment significantly changed the gut microbiota composition in mice fed an HFD. The richness and diversity of the gut microbiota were notably decreased upon wild rice consumption. Compared with a normal chow diet (NCD), HFD feeding altered 117 operational taxonomic units (OTUs), and wild rice supplementation reversed 90 OTUs to the configuration in the NCD group. Overall, our results suggest that wild rice may be used as a probiotic agent to reverse HFD-induced MAFLD through the modulation of the gut microbiota.
\end{abstract}

Keywords: gut microbiota; inflammation; insulin resistance; nonalcoholic fatty liver disease; wild rice

\section{Introduction}

With the improvement of social living standards, the daily diet structure of people has undergone a tremendous change from plant-based foods to high-fat and low-carb carbohydrates. Long-term excessive high-fat intake could induce obesity, which has been linked with major degenerative diseases and the development of metabolic (dysfunction) associated fatty liver disease (MAFLD), formerly known as non-alcoholic fatty liver disease (NAFLD) [1,2]. MAFLD affects about $25 \%$ of the global population and is associated with metabolic derangements such as visceral obesity, increased fasting blood glucose, dyslipidemia, and hypertension [2]. Within the global MAFLD population, around $60 \%$ and $40 \%$ of the population was classified as obese and non-obese, respectively [3]. Moreover, the incidence of MAFLD varies worldwide, and is relatively high in developed countries such as Europe and the United States [4,5]. In developing countries, the incidence of the disease is about 15\%, but in obese patients, the incidence is as high as $50 \%$ or more [4]. It has been shown that MAFLD is not associated with liver-related disease, but increasing research has demonstrated that MAFLD is linked with various chronic diseases [6]. For example, MAFLD can increase the risk of hypertension, type 2 diabetes mellitus, oxidative stress, chronic kidney disease, etc., which all threaten human health [6-8]. MAFLD and its associated metabolic syndrome have become a global concern. 
Growing evidence indicates that MAFLD and metabolic syndrome can influence the gut microbiota compositions [9]. The gut microbiota is a diverse ecosystem composed of bacteria, archaea, fungi, and viruses. Existing studies have found that patients with obese and non-obese MAFLD have an imbalance of gut microbiota [10-12]. For example, there was an increased abundance of the species Dorea and a reduction in the relative abundance of a number of species, including Marvinbryantia and the Cbristensellenaceae R7 group in patients with non-obese MAFLD [13]. Interestingly, mice fed the cholesterol-rich diet were observed to have an increased relative abundance of Bacteroidetes and a decrease in Firmicutes compared with those fed with high sucrose, suggesting a clearly differential profile of gut microbiota between obese and non-obese subjects with MAFLD [13]. Recent reports have shown that the gut microbiota may promote the progress of MAFLD through the intestinal-hepatic axis pathway [14]. More specifically, increased intestinal permeability and bacterial translocation can allow intestinal microbes and metabolites to reach the liver, thereby affecting bile acid metabolism, and promoting intestinal motility disorders and systemic inflammation [14]. Furthermore, the gut microbiota can contribute to the occurrence and development of MAFLD by regulating energy balance, increasing the synthesis of short chain fatty acids (SCAs) and triglycerides, regulating choline metabolism and bile acid balance, producing endogenous ethanol, bacteria-derived toxins, and promoting the release of pro-inflammatory cytokines in the liver [15-17].

Despite tremendous progress in the research on the pathogenesis of MAFLD, an effective therapeutic method is still being actively explored. A large number of studies have shown that changing the diet structure can help patients with non-alcoholic fatty liver disease improve their condition. Wild rice (WR) is a genus of grasses (Zizania), but not a type of rice. There are four species of WR belonging to the genus Zizania. Zizania aquatica L., Zizania palustris L., and Zizania texana Hitche are native to North America, whereas Zizania latifolia (Griseb) Turcz is native to China, Japan, and Vietnam [18]. Wild rice is now considered a functional food, and is currently available in grocery stores in North America because of several of its characters, such as low contents in cholesterol and its abilities in anti-inflammatory and antioxidant aspects [18-20]. Because WR has a chewy dark outer layer and soft inner grain after cooking, it has been used as a component of salads or steamed mixed grains. Numerous reports have shown that WR has a higher content of protein; dietary fiber; vitamin B1, B2, E; and minerals than common white rice [20-22]. Moreover, the contents of phenolic acids and flavonoids in wild rice are significantly higher than that in white rice $[23,24]$. Plenty of research suggests proanthocyanidins plays a key role in changing the composition of the gut microbiota based on in vitro and in vivo models and the bioactivities of their metabolites [25-27]. However, it has not been reported that consumption of WR could prevent MAFLD via the modulation of the gut microbiota. Therefore, we assessed the effect of WR on nonalcoholic fatty liver disease induced by a high-fat diet. Furthermore, we found that WR causes substantial changes in the gut microbiota composition, suggesting WR has attenuated many of the adverse health consequences associated with an HFD, and it modulates the gut microbiota in a positive way.

\section{Results}

\subsection{General Nutrition Composition}

The protein contents of the wild rice samples $(15.6 \pm 0.21 \mathrm{~g} / 100 \mathrm{~g})$ were markedly higher than that of the white rice $(10.19 \pm 0.29 \mathrm{~g} / 100 \mathrm{~g})$ and red rice $(11.78 \pm 0.11 \mathrm{~g} / 100 \mathrm{~g}$; Table 1$)$. The average fat and ash content of the wild rice was similar to red rice, and was 1.67 and 4.36 times as high as that of white rice, respectively. The moisture was about $10 \%$ in all of the rice samples, and less than that in white rice. The sodium content, dietary fiber, resistant starch, and total phenolic in wild rice exceeded those of white rice and red rice. Wild rice is a caryopsis with a seed coat that contains a lot of minerals and dietary fiber. Hence, wild rice is considered to be a possible food for people with diabetes. 
Table 1. Comparison of general nutrition composition among wild rice, white rice, and red rice (mean \pm standard deviation (SD)).

\begin{tabular}{cccc}
\hline Composition & White Rice $(\mathbf{g} / \mathbf{1 0 0} \mathbf{g})$ & Red Rice $(\mathbf{g} / \mathbf{1 0 0} \mathbf{g})$ & Wild Rice $(\mathbf{g} / \mathbf{1 0 0} \mathbf{g})$ \\
\hline Crude protein & $10.19 \pm 0.29^{\mathrm{a}}$ & $11.78 \pm 0.11^{\mathrm{a}}$ & $15.6 \pm 0.21^{\mathrm{b}}$ \\
Total fat & $0.67 \pm 0.01^{\mathrm{a}}$ & $1.36 \pm 0.05^{\mathrm{b}}$ & $1.12 \pm 0.01^{\mathrm{b}}$ \\
Moisture & $11.76 \pm 0.10^{\mathrm{a}}$ & $10.61 \pm 0.17^{\mathrm{a}}$ & $10.16 \pm 0.12^{\mathrm{a}}$ \\
Total ash & $0.30 \pm 0.02^{\mathrm{a}}$ & $1.38 \pm 0.02^{\mathrm{b}}$ & $1.31 \pm 0.25^{\mathrm{b}}$ \\
Sodium & $11.8 \pm 0.87^{\mathrm{a}}$ & $84.5 \pm 4.55^{\mathrm{b}}$ & $5.21 \pm 0.61^{\mathrm{c}}$ \\
Dietary fiber & $0.42 \pm 0.01^{\mathrm{a}}$ & $2.68 \pm 0.01^{\mathrm{b}}$ & $6.83 \pm 0.11^{\mathrm{c}}$ \\
Resistant starch & $1.41 \pm 0.04$ & $0.95 \pm 0.04^{\mathrm{a}}$ & $10.87 \pm 0.15$ \\
Total phenolic [25] & $1.30 \pm 0.00^{\mathrm{a}}$ & $1.40 \pm 0.00^{\mathrm{a}}$ & $2.10 \pm 0.00^{\mathrm{b}}$ \\
\hline
\end{tabular}

$a, b, c:$ The values of each group with the same letters are not significantly different in the analysis of variance followed by Tukey's post-hoc test ( $n=5$ per group).

\subsection{WR Attenuates Features of Obesity in HFD-Fed Mice}

Compared with the NCD mice, the HFD-fed mice gained more weight (Figure 1A) and developed hallmark features of hyperlipidemia, including serum lipid profiles (increased serum TG (Figure 1B), TC (Figure 1C), LDL (Figure 1D), and decreased serum HDL (Figure 1E)), hepatic lipid profiles (liver TG (Figure 1F), TC (Figure 1G), LDL (Figure 1H), and decreased liver HDL (Figure 1I)), increased impaired glucose clearance in the oral glucose tolerance test (OGTT; Figure 1J,K), and insulin resistance (Figure 1L,M). Treatments with WR significantly attenuated HFD-induced weight gain (Figure 1A). The attenuated weight gain was associated with modestly reduced serum and liver TG (Figure 1B,F), TC (Figure 1C,G), and LDL (Figure 1D,H), as well as increased serum and liver HDL (Figure 1E,I). In addition, dietary supplementation with WR significantly increased the clearance of a glucose bolus during the OGTT (Figure 1J,K) and improved HFD-induced insulin resistance (Figure 1L,M) Thus, WR enhanced the glucose-insulin homeostasis by attenuating HFD-induced MAFLD.

\subsection{WR Prevents HFD-Induced Liver Steatosis, Oxidative Stress, and Systemic Low-Grade Inflammation}

Next, the liver steatosis was evaluated by HE staining and transmission electron microscopy. The HFD group showed representative characteristics of liver steatosis (Figure 2A,B), such as a large number of lipid droplet vacuoles, irregular arrangement of cells, and cytoplasm rarefaction, an effect that was ameliorated by the WR treatment (Figure 2A,B). Previous studies have shown that obesity and liver steatosis are associated with liver oxidative stress. We evaluated the effects of WR on liver oxidative stress by examining the level of SOD, GSH-Px, and MDA. The SOD (Figure 2C) and GSH-Px (Figure 2D) levels in the HFD mice were significantly lower than in the NCD mice, and the MDA content (Figure 2E) was significantly higher than in the control group. As expected, an HFD supplemented with WR significantly increased the SOD and GSH-Px content (Figure 2C,D), and reduced the MDA content compared with the HFD mice (Figure 2E). Previous studies have shown that liver oxidative stress is associated with low-grade chronic inflammation. We investigated the effects of WR on the serum inflammatory cytokine (IL-1, IL-6, and IL-8) levels. WR treatment reduced systemic low-grade inflammation in HFD-fed obese mice (Figure $2 \mathrm{~F}-\mathrm{H}$ ). Because NF- $\mathrm{KB}$ is a key player in inflammatory reactions, HFD feeding significantly enhanced the mRNA and protein levels of NF-KB (Figure 2I,K), and reduced the mRNA levels of IKB- $\alpha$ (Figure 2J,K) in the liver, whereas WR supplementation restored their levels (Figure 2I-K). 


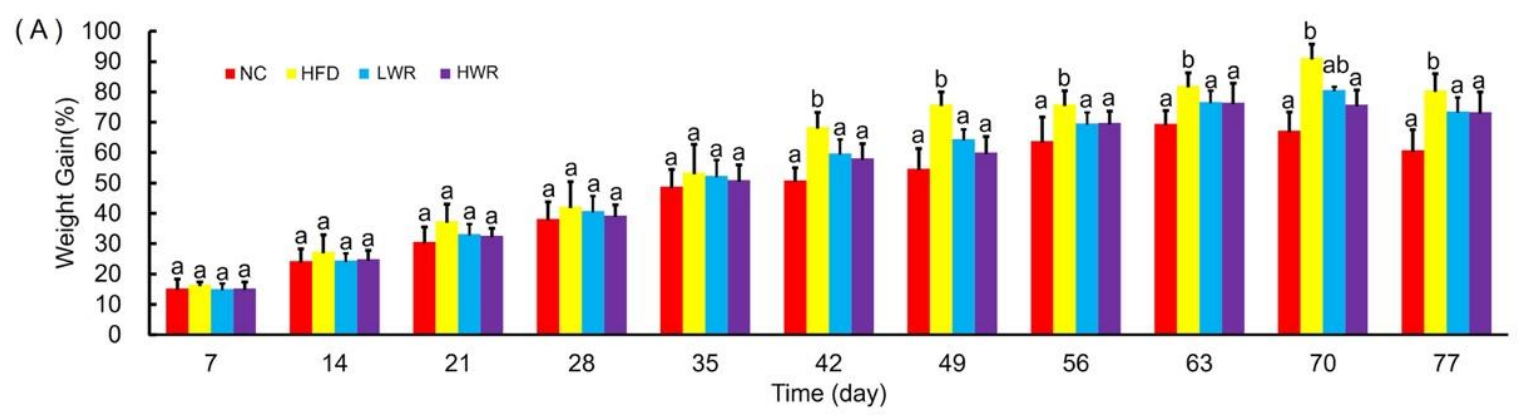

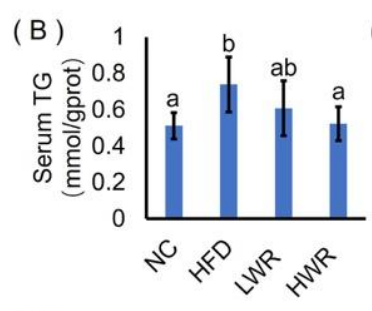

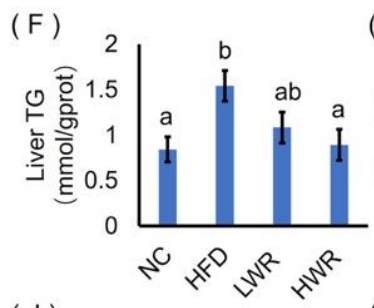

( J )

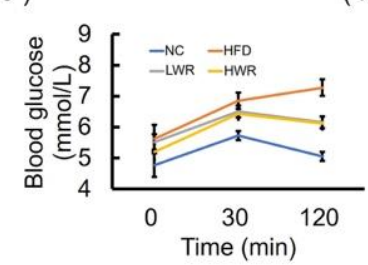

(C) 2

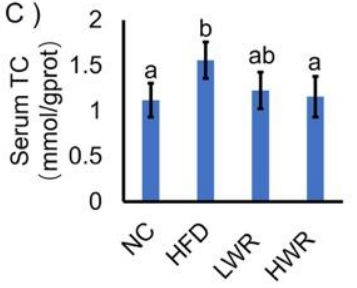

( $G$ )

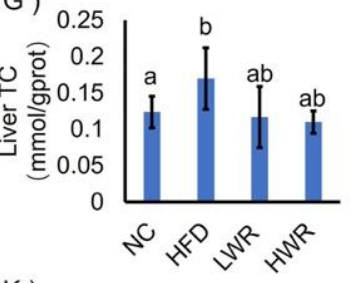

( K)

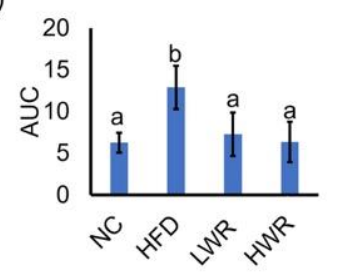

( D )

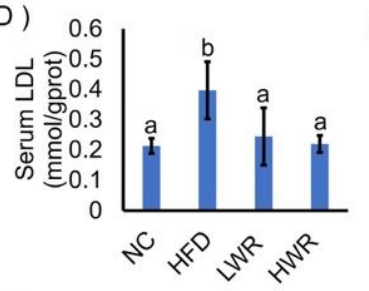

( $\mathrm{E}$ ) $\quad 0.8$

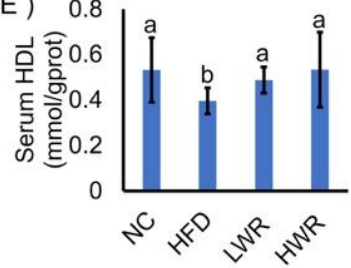

( $\mathrm{H}$ )

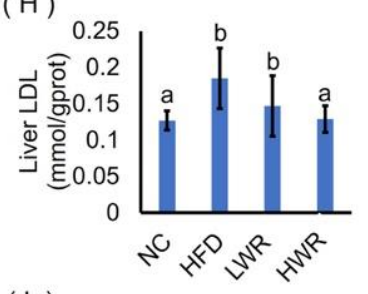

(1)

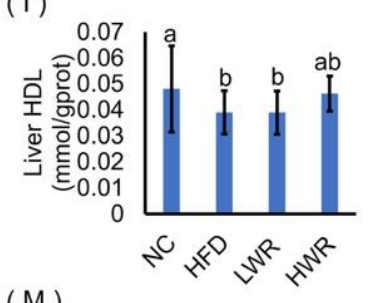

( L)

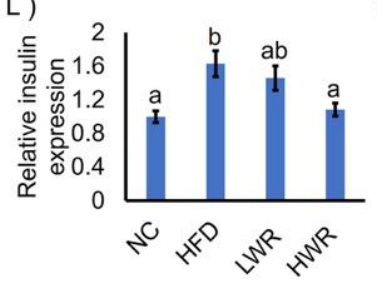

( $\mathrm{M}$ )

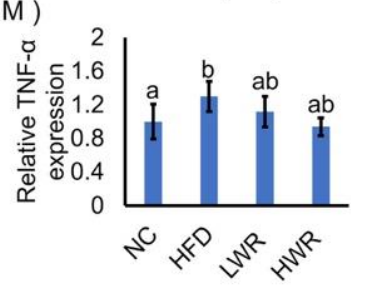

Figure 1. Wild rice attenuated high-fat diet (HFD)-induced obesity and impaired glucose-insulin homeostasis. (A) Body weight gain as a percentage of baseline weight for each mouse. (B) Serum concentrations of triacylglycerol (TG). (C) Serum concentrations of total cholesterol (TC). (D) Serum concentrations of low-density lipoprotein (LDL). (E) Serum concentrations of high-density lipoprotein (HDL). (F) Liver concentrations of triacylglycerol (TG). (G) Liver concentrations of total cholesterol (TC). (H) Liver concentrations of low-density lipoprotein (LDL). (I) Liver concentrations of high-density lipoprotein (HDL). (J) Curve of oral glucose tolerance test (OGTT). (K) Areas under the curve (AUC) of OGTT. (L) Serum relative expression of insulin (INS). (M) Liver relative expression of tumor necrosis factor- $\alpha$ (TNF- $\alpha$ ). NC presents normal chow diet group. HFD presents high-fat diet group. LWR presents low dose wild rice diet group. HWR present high dose wild rice diet group. a,ab,b: The values of each group with the same letters are not significantly different in the analysis of variance followed by Tukey's post-hoc test ( $n=8$ mice per group).

\subsection{WR Alters the Gut Microbiota Composition in HFD-Induced Mice}

To investigate the effect of WR on the gut microbiota composition in HFD-induced mice, we amplified and sequenced the V3 + V4 region of the 16S rRNA of each sample. Finally, we obtained 1188756 clean tags, which were used for the following analysis (Table S3). The alpha diversity analysis showed that the HFD-fed mice treated with WR showed significantly reductions in the richness and diversities of their gut microbiota based on the numbers of OTUs (Figure 3A), rarefaction curves (Figure 3B), Chao1 curves (Figure 3C) and index of Chao1 (Figure 3D), index of Observed species (Figure 3E), index of PD whole tree (Figure 3F), and index of goods coverage (Figure 3G). Furthermore, we also found that HFD feeding significantly decreased the Bacteroidetes abundance, while it increased 
the abundance of Firmicutes. In contrast, WR recovered these levels and affected the ratio of Firmicutes to Bacteroidetes (Figure 3H). In addition, we found that the results of the genus level and phylum level analysis were consistent. For example, WR significantly increased the relative abundance of Lactobacillus in HFD-fed mice (Figure 3I). In addition, according to the analysis results of NMDS, PCoA, and PCA, the structure of the WR treatment group was similar to that of NCD group when comparing these indexes with the HFD group. (Figure 3J-L).
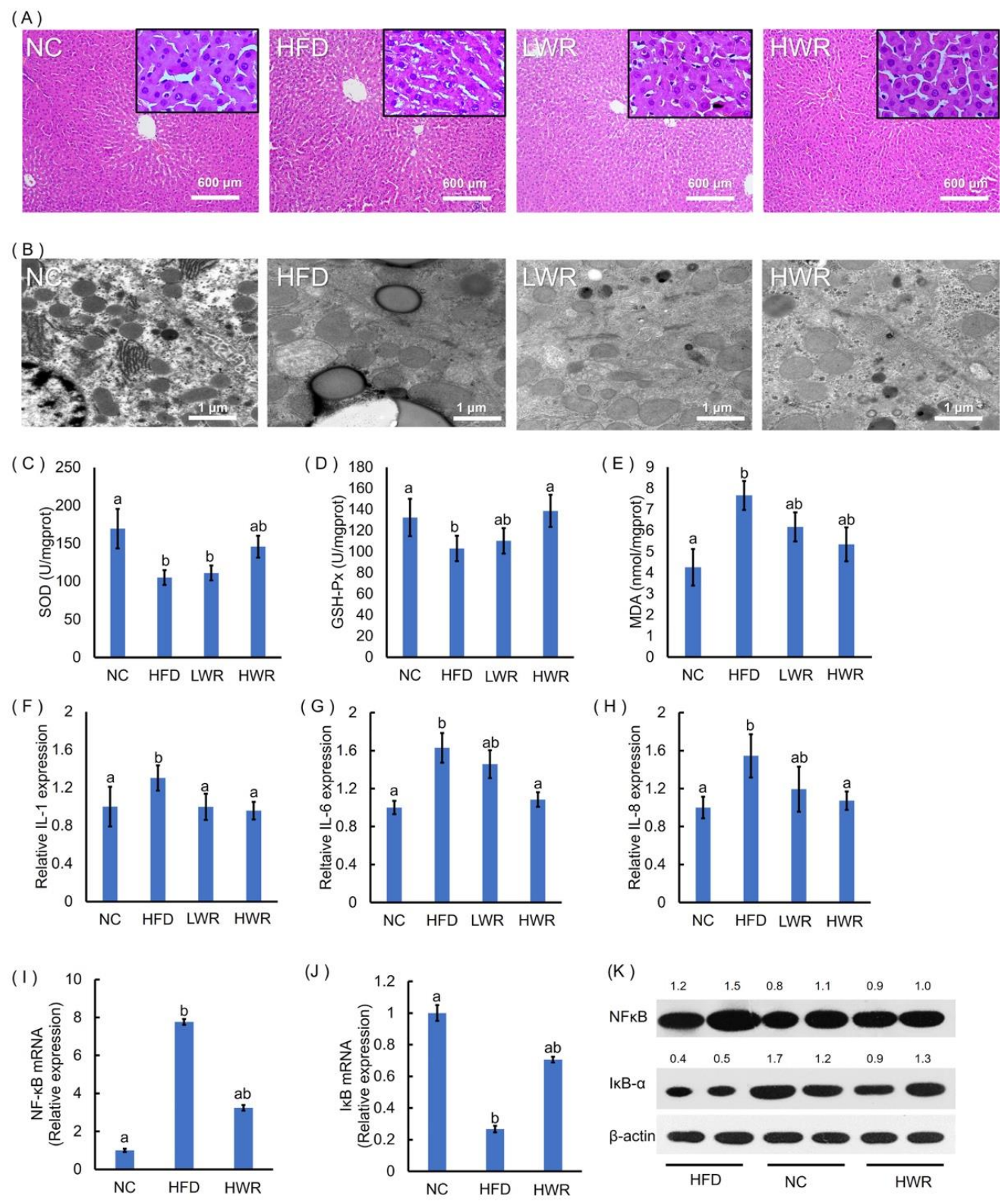

Figure 2. Wild rice prevents HFD-induced adipocyte hypertrophy, hepatic steatosis, and systemic inflammation. (A) Hematoxylin and eosin (HE)-stained liver sections. (B) Ultrastructural changes in the liver were detected via TEM. The (C) liver superoxide dismutase (SOD), (D) GSH-Px, and (E) malondialdehyde (MDA) were measured using commercial assay kits. The (F) interleukin (IL)-1, (G) IL-6, and (H) IL-8 concentrations were measured using ELISA. Gene expression levels of (I) NFkB and (J) IKB- $\alpha$ in the liver were measured by qRT-PCR. (K) Liver NFKB and IKB- $\alpha$ protein production was examined by Western blot, and relative protein levels were normalized with $\beta$-actin $(n=3)$. $(\mathbf{C}-\mathbf{J}) \mathrm{a}, \mathrm{ab}, \mathrm{b}$ : The values of each group with the same letters are not significantly different in the analysis of variance followed by Tukey's post-hoc test. 

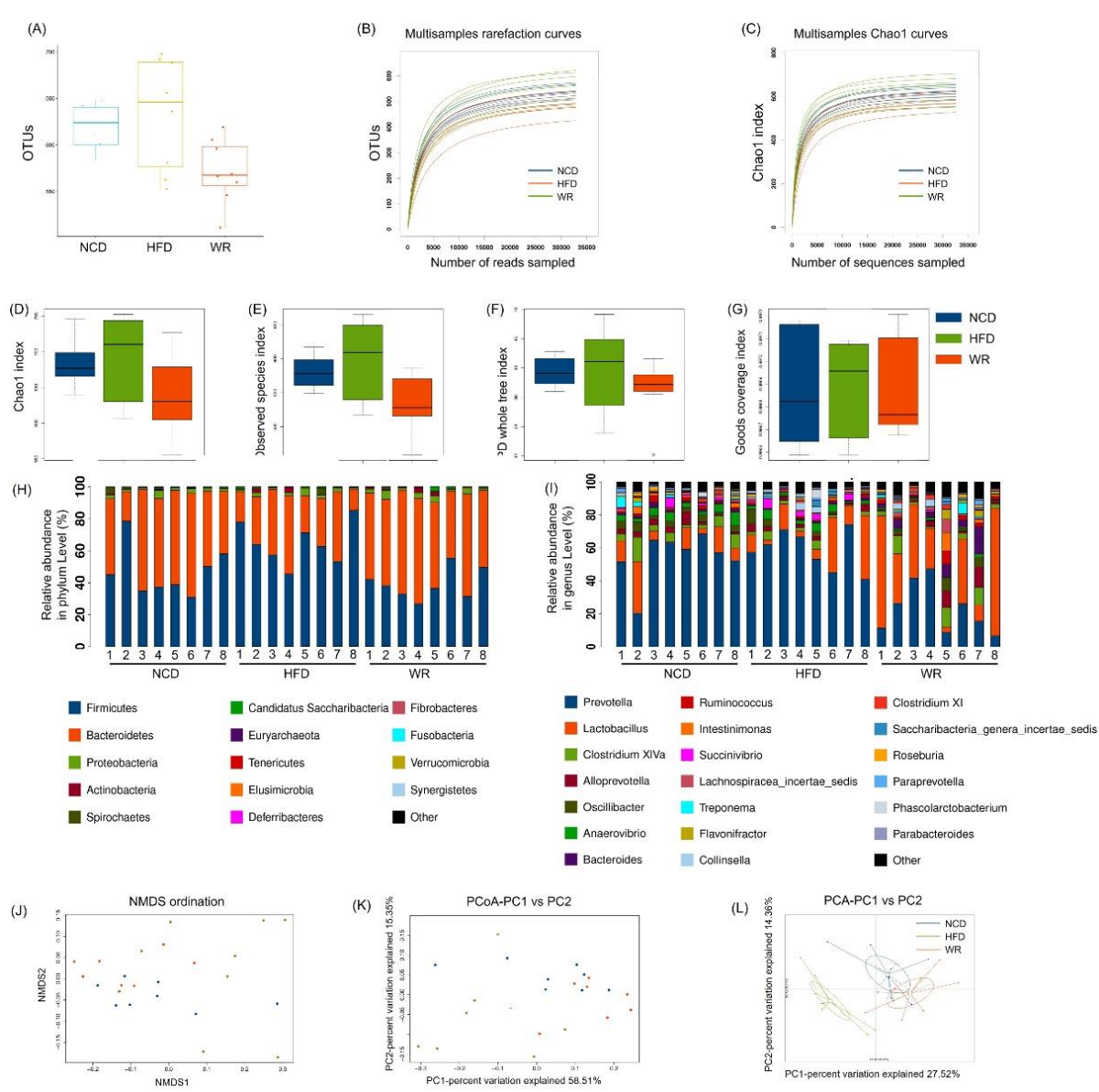

Figure 3. Comparison of the alpha diversities, species richness, and structures of the gut microbiota between wild rice and HFE-fed mice. (A) The numbers of OTUs. (B) Rarefaction curves and (C) Chao1 curves. (D-G) Index of the Chao1 index, observed species, phylogenetic diversity (PD) whole tree, and goods coverage of each group, respectively. All of the values are presented as mean $\pm \operatorname{SE}(n=5)$. Relative abundances at the level of the $(\mathbf{H})$ phylum and (I) genus level. (J-L) NMDS, PCoA, and PCA of each sample. NCD presents normal chow diet group. HFD presents high-fat diet group. WR presents wild rice diet group, respectively.

\subsection{WR Modulates the Key Phylotypes of Gut Microbiota in HFD-Fed Mice}

In order to explore the microbiology communities induced by WR, we analyzed their effective sequences via redundancy analysis (RDA). In total, 136 predictive OTUs were identified (Figure 4A,B and Table S1). Among these OTUs, HFD feeding altered 117 OTUs (50 increased and 67 decreased) compared with the NCD group. However, the treatment of WR could generate 90 variable OUTs, among which 70 increased and 20 decreased. Furthermore, we found that 71 OTUs showed reverse correction in the HFD-fed and NCD groups. For example, Ruminococcaceae, Desulfovibrionaceae, Bacteroides, and Porphyromonadaceae have been negatively correlated with WR. We compared the gut microbiota of different treatments using LEfSe to identify the specific bacterial taxa associated with WR treatment. The LEfSe analysis showed that WR increased Lactobacillus (Figures 4 and 5A), which is a probiotic that helps reduce obesity, diabetes, and inflammation caused by HFD [28-30], while it decreased the negative microorganisms such as Prevotella, Bacteroides, and Staphylococcus (Figures 4 and 5B-D). What is more, we found that some known bacteria (such as Prevotella, Bacteroides, and Staphylococcus) with negative effects were observed with decreased trends after treatment in the HFD-fed mice with WR (Figures 4 and 5B-D). These results demonstrate that WR treatment reverses some of the changes in the gut microbiota composition induced by a HFD. 


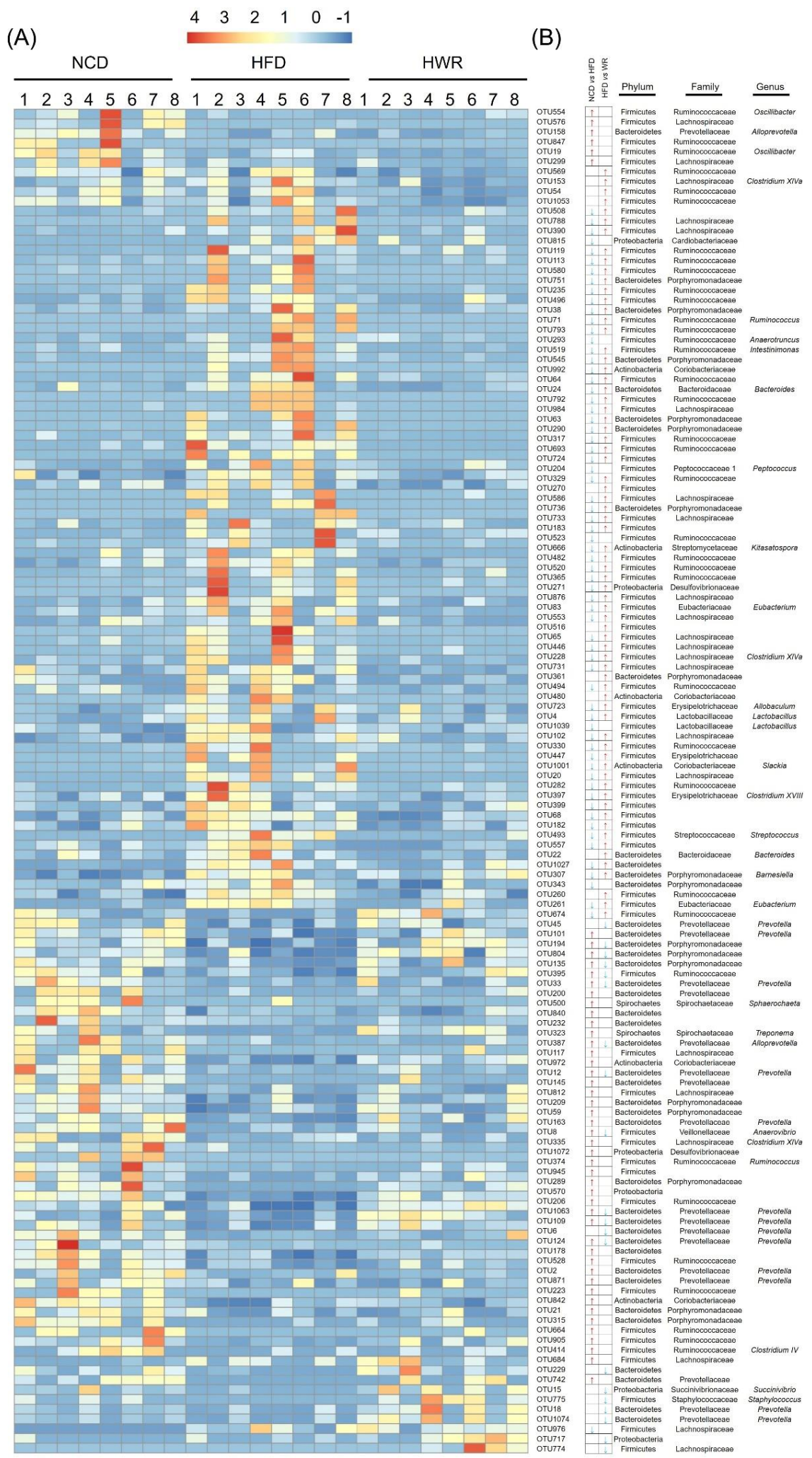

Figure 4. Heatmap and representative bacterial statistics of redundancy analysis (RDA)-identified OTUs and LefSe among differentially taxons in the normal chow diet (NCD) group and experimental groups. (A) Heat map of the relative abundance of RDA-identified key 136 OTUs. (B) Statistics of representative bacterial taxons at the genus, family, and phylum levels based on OTUs. The red and blue arrows represent the increased or decreased OTUs in the normal chow diet (NCD) and HWR groups relative to the HFD-fed group $(p<0.01)$. 
( A )

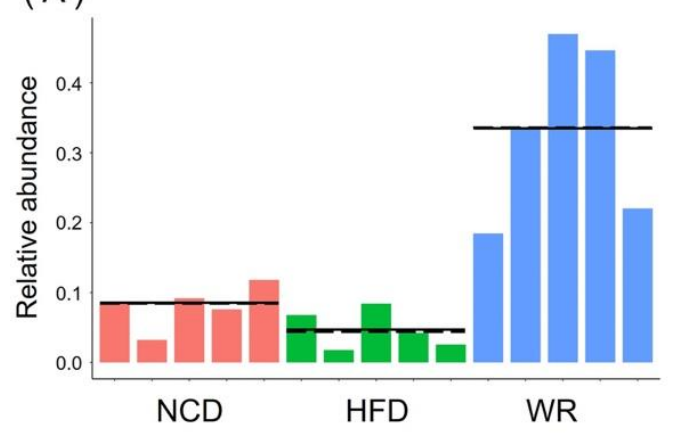

( C )

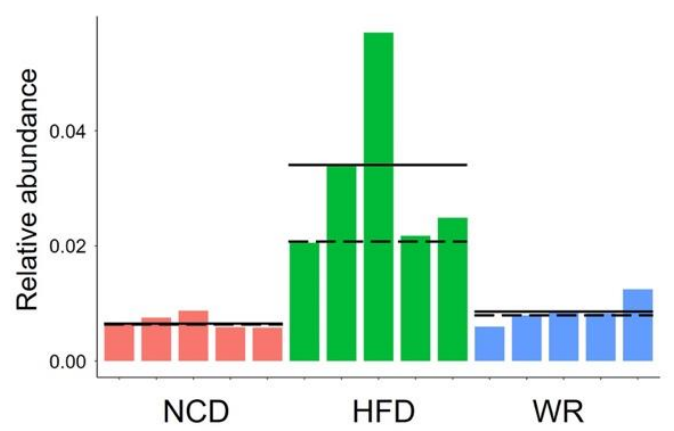

( B )

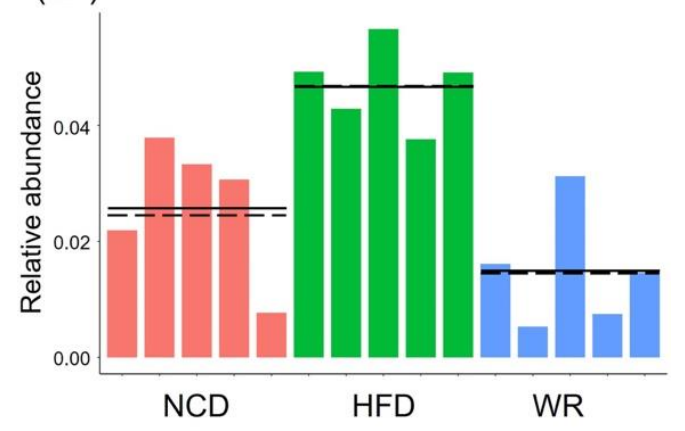

( D )

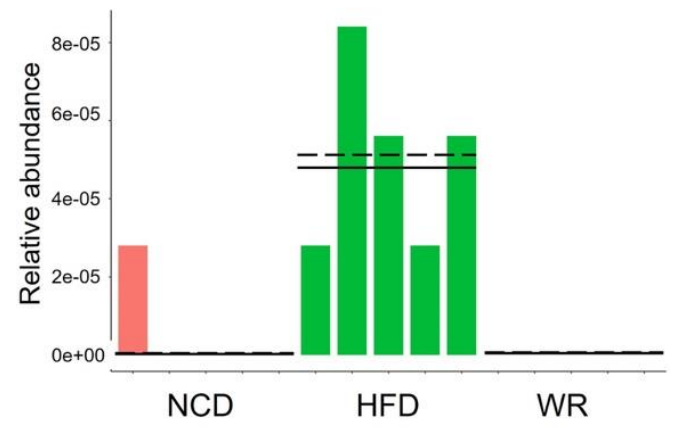

Figure 5. The relative abundance of (A) Lactobacillus, (B) Prevotella, (C) Anaerovibrio, and (D) Staphylococcus obtained in fecal microbiota from the LefSe results. Solid and dashed lines indicate the mean and median, respectively.

\section{Discussion}

The gut microbiota is closely correlated with MAFLD and inflammation. Although several studies have shown that wild rice has anti-MAFLD and anti-inflammatory activities in different animal models [18,31-33], the effects of wild rice on the gut microbiota have not yet been investigated. Herein, we provide evidence that wild rice oral administration for 11 weeks has a protective effect against dietary-induced MAFLD, insulin resistance, liver steatosis, and low-grade inflammation via the modulation of the gut microbiota. As already mentioned, the gut microbiota of MAFLD humans and rodent models is highly associated with decreased abundances of Bacteroidetes and increased abundances of Firmicutes [34-36]. Our results show that wild rice supplementation significantly increased the abundance of Bacteroidetes and decreased the abundance of Firmicutes in HFD-fed mice (Figure 3E,F). The effects could be attributed to the high dietary fiber, resistant starch, and polyphenol content of WD. In addition, the abundance of Gram-negative bacteria, such as Prevotellaceae, was increased in the intestinal ecosystem of HFD mice and decreased in WR mice. These effects may be due to the total phenolic in wild rice. Many studies have revealed the effects of proanthocyanidins from different sources against gut microbiota, including Prevotella [27,37]. Numerous reports revealed that Prevotellaceae and the included genera are associated with low-grade inflammation [38-40].

Additionally, our results demonstrate that wild rice significantly enhanced the level of Lactobacillus (Figures 3I and 4, Table S1). Preliminary reports demonstrated HFD, to a greater or lesser degree, decreased the relative abundance of Lactobacillus, a probiotic belonging to the Lactobacillaceae family, which is associated with nutrient metabolism [41]. In detail, Khare et al. explored the effect of cinnamaldehyde in HFD mice, and found that HFD feeding can decrease the abundance of Lactobacillus species [42]. Furthermore, the abundance of Lactobacillus showed a decreasing tendency when investigating the effect of $\alpha$-cyclodextrin on the gut microbiota in HFD mice [43]. Similarly, the microbiological analysis of the gut microbiotas in HFD mice showed that the abundance of Lactobacillus decreased, while it increased when treated with the pandanus tectorius fruit extract and isomalto-oligosaccharides [44,45]. Moreover, Bacteroides, a genus in the family Bacteroidetes, was associated with increases in the acetic acid and propionic acid levels in the gut, 
and its abundance was also restored by wild rice in the HFD-fed mice (Figure 4), which is consistent with previous findings [46,47]. Prevotella predominantly stimulates cells to produce IL-1, IL-6, and IL-8, resulting in metabolic disorders and low-grade systemic inflammation [48]. The level of Prevotella was reduced by wild rice in HFD-fed mice in the present study (Figures 4 and 5B).

Recent studies have shown an interrelation between oxidative stress and low-grade inflammation in humans and rodents [49]. Our research demonstrated that the antioxidant status of HFD-fed mice was significantly improved by treatement with WR, characterized by an increased content of MDA and decreased activities of GSH-Px and SOD (Figure 2C-E), which is consistent with previous research [18]. It has also been reported that there is a relationship between obesity and low-grade inflammation. Our study showed similar results with a previous study, that the accumulation of hepatic lipids induced by HFD was significantly reduced in the treatment group (Figure 1F-I and Figure 2A,B) [18]. In addition to the changes in the physiological indicators, it has been shown that the NF- $\mathrm{kB}$ signaling pathway participated in the processes of inflammation and diabetes [50]. In the WR-treated groups, all markers of systemic low-grade inflammation, such as TNF- $\alpha$, IL-1, IL-6, IL-8, and NF-kB, exhibited downregulation, whereas IKB- $\alpha$ was induced (Figures $1 \mathrm{M}$ and $2 \mathrm{~F}-\mathrm{K}$ ). Additionally, the circulating TG, $\mathrm{TC}$, blood glucose, and insulin levels increased in the HFD-fed mice. Wild rice, on the other hand, protected against increased insulin resistance in obese mice (Figure 1). The purpose of this study was to unveil the potential influence of the wild rice on the gut microbiota and the NF- $\mathrm{kB}$ signaling pathway. Further studies are still needed in order to elucidate its mechanism.

\section{Materials and Methods}

\subsection{Diet Preparation}

Whole grains of wild rice (Z. latifolia) were hand-harvested from Baimahu Village, Qianfeng Town, Jinhu County, Huai'an City, Jiangsu Province, China ( $33^{\circ} 11^{\prime} 9^{\prime \prime}$ N; 119 $9^{\circ} 37^{\prime \prime}$ E) on 20 September 2017. After air drying, full and plump seeds were picked and ground to a fine powder in a mechanical grinder, sieved through a $0.45 \mathrm{~mm}$ sifter, and maintained in a desiccator until use. The seeds and experimental diets were sterilized by $\gamma$ irradiation at $10 \mathrm{kGy}$. The normal chow diet (NCD) was purchased from Beijing HFK Bioscience Co., Ltd. (Beijing, China).

\subsection{Proximate Composition Analysis}

The proximate compositions of the crude protein, total lipid, moisture, total ash, sodium $(\mathrm{Na})$, dietary fiber, and $t$ resistant starch of all of the samples were analyzed according to the Chinese National Standards, namely: GB 5009.5-2016, GB 5009.6-2016, GB 5009.3-2016, GB 5009.4-2016, GB 5009.91-2016, GB/Z 21922-2008 2.2.8, GB28050-2011, GB 5009.88-2014, and NY/T 2638-2014, respectively.

\subsection{Animal Trial}

Ten-week-old male C57BL/6J mice were obtained from Shandong Experimental Animal Center (Jinan, China). All of the mice were fed an identical diet of normal chow during the first two weeks. After this period, the mice were randomly divided into four dietary groups (six mice per cage, two cages per group) in a room in a room at $25 \pm 2{ }^{\circ} \mathrm{C}$ and $55 \pm 5 \%$ relative humidity with controlled lighting (12:12 $\mathrm{h}$ light/dark cycle), with free access to water and the appropriate diets. The NCD group received the normal rodent chow, the HFD group received the high-fat diet (normal chow containing $10 \%$ lard), and the wild rice diet groups received normal chow containing $10 \%$ lard $+10 \%$ wild rice or $20 \%$ wild rice for 11 weeks. The compositions of the experimental diets are shown in Table S1. The food intake was recorded daily, and the body weight was recorded every week. All efforts were made to reduce animal suffering. Experimental procedures were performed according to the guidelines of the Ethics and Animal Welfare Committee of Shandong Academy of Medical Sciences (Jinan, China, SCXK-20140007). All of the sections of this report adhere to the ARRIVE Guidelines for reporting animal research [51]. 


\subsection{Biochemical Analysis}

Blood samples were collected from fasted mice at the end of the eleventh week. The serum was separated by centrifuging the samples at $2000 \times g$ for $10 \mathrm{~min}$ at $4{ }^{\circ} \mathrm{C}$, and was then stored in sterile Eppendorf tubes at $80^{\circ} \mathrm{C}$. The livers were carefully removed, cut into several sections, and then stored at $-80^{\circ} \mathrm{C}$ after snap-freezing in liquid nitrogen. A total of $0.1 \mathrm{~g}$ liver tissue was homogenized with $0.9 \mathrm{~mL}$ of saline using a homogenizer (Biospec Products, Bartlesville, OK, USA). The liver homogenate was centrifuged at $1800 \times g$ at $480{ }^{\circ} \mathrm{C}$ for $15 \mathrm{~min}$, and the supernatant was subjected to further analysis. Serum parameters including total cholesterol (TC), triglycerides (TG), low density lipoprotein (LDL), and high density lipoprotein (HDL) levels, as well as liver parameters, including TC, TG, LDL, HDL, interleukin-1(IL-1), IL-6, IL-8, rat tumor necrosis factor alpha (TNF- $\alpha$ ), insulin, superoxide dismutase (SOD), malondialdehyde (MDA), and glutathione peroxidase (GSH-px) levels, were determined using analytical reagent kits (Nanjing Jiancheng Bioengineering Institute, Nanjing, Jiangsu, China). A microplate reader (Multiskan GO, Thermo Fisher Scientific, Waltham, MA, USA) was used to measure the kinetic UV. Each assay was performed at least three times.

\subsection{Oral Glucose Tolerance Tests}

For the oral glucose tolerance tests, during the eighth week of the experiment, the mice were fasted for $12 \mathrm{~h}$ before receiving an oral administration of D-glucose $(1.5 \mathrm{~g} / \mathrm{kg})$. Blood samples were taken from the tail vein at 30 and $120 \mathrm{~min}$ following glucose administration, and the blood glucose was measured using a glucometer (Johnson and Johnson, Shanghai, China).

\subsection{Liver Histopathology}

Approximately $8-\mu \mathrm{m}$-thick frozen liver sections were prepared to measure the accumulation of hepatic fat. The histopathological changes in the frozen liver sections of the mice were examined using HE staining, as previously described [52].

\subsection{Liver Ultrastructure Examination}

The liver ultrastructure was investigated using transmission electron microscopy (TEM), as described previously [53]. Briefly, livers were collected, as mentioned above, and fixed with $2 \%$ glutaraldehyde (containing $0.1 \mathrm{M}$ sucrose and $0.2 \mathrm{M}$ sodium cacodylate) overnight at $4{ }^{\circ} \mathrm{C}$, followed by $10 \mathrm{~g} / \mathrm{L}$ osmium tetroxide. The samples were dehydrated and embedded in epoxy resin. Ultra-thin sections were observed using an H700 TEM (Hitachi, Tokyo, Japan).

\subsection{Western Blot Analysis}

The liver protein was extracted using radio immunoprecipitation assay (RIPA) lysis. The protein amount was measured using a BCA protein assay reagent from Pierce (Rockford, IL, USA). The protein samples were separated by $10 \%$ SDS-PAGE gel and transferred onto Immobilon ${ }^{\circledR}$-P transfer membranes (Sigma-Aldrich, St. Louis, MO, USA). After blocking with $8 \%$ nonfat milk, the membranes were first incubated with primary antibodies against nuclear factor (NF)- KB (1:2000; Cell Signaling Technology, Danvers, MA, USA), IкB- $\alpha$ (1:1000; Cell Signaling Technology), and $\beta$-actin (1:6000; Cell Signaling Technology) at $4{ }^{\circ} \mathrm{C}$ for $12 \mathrm{~h}$, and then with the secondary antibody for $1 \mathrm{~h}$, as described previously [54]. The intensities of the protein bands were quantified with Image J software (http://imagej.nih.gov/ij/) and the values normalized to $\beta$-actin.

\subsection{Gene Expression Analysis}

The tissue total RNA was isolated with an RNAprep Pure tissue kit from Tiangen Biotech Co., Ltd. (Beijing, China). For real-time PCR analysis, reverse transcription was performed with oligdT-18 and M-MLV transcriptase from Promega (Madison, WI, USA). Real-time PCR was performed using SYBR Green qPCR Super Mix (Invitrogen, San Diego, CA, USA) on an ABI 7500 real-time PCR 
system (Applied Biosystems, Foster City, CA, USA), according to the manufacturer's instructions, as described previously. The values were normalized against the control glyceraldehyde-3- phosphate dehydrogenase (GAPDH). The sequences of the real-time PCR primers used are listed in Table S2.

\subsection{Gut Microbiota Analysis}

On the last day of the animal trial for the gut microbial analysis, we collected the fresh fecal samples and then extracted the total DNA following the manufacturer's recommendations. After measuring the concentration of DNA using a Nanodrop 8000 spectrophotometer (Thermo Fisher Scientific), the DNA sample was diluted with sterile water to a concentration of $20 \mathrm{ng} / \mathrm{L}$. The sequencing library of the bacterial 16S rRNA gene V3-V4 region in the DNA samples was constructed by PCR using bacterial primers (Table S2), according to the modified guide provided by Illumina (Part \# 15044223 Rev. B). The raw data were firstly merged with FLASH [55] and then separated based on their unique sequencing barcodes. All high-quality sequencing sequences were merged, and the effective sequences were obtained after removing the exact same sequences. The effective sequences were clustered into operational taxonomic units (OTUs) according to their sequence similarities (above 97\%) [56]. Based on the Quantitative Insight IntoMicrobial Ecology, v. 1.8.0 (QIIME) platform, the alpha diversity analysis and beta-diversity analysis were analyzed [57-59]. The total OTU number, Shannon diversity index, and Faith's phylogenetic diversity (PD whole tree) were used to indicate the alpha diversity of each sample, and the Mann-Whitney U test was used in GraphPad Prism 7 software to analyze each group. Multi values such as the OTU rank curves, Shannon index, calculated indexes of Chao1, PD whole tree index, and goods coverage were used for calculating their alpha diversities. Meanwhile, we assessed the beta diversities based on nonmetric multidimensional scaling (NMDS), uniFrac distance-based principal coordinate analysis (PCoA), and principal component analysis (PCA), together with RDA-identified key OTUs.

The total OTU number, Shannon diversity index, and Faith's phylogenetic diversity (PD Whole tree) were used to indicate the alpha diversity of each sample, and the Mann-Whitney U test was used in GraphPad Prism 7 software to analyze in each group the significance of the difference. Beta diversity analysis was mainly based on the principal coordinate analysis (PCoA), based on the Bray-Curtis distance and the normalized sample-OTU abundance matrix. The obtained PCoA score map can visually show the small groups in the 10th week of intervention. The dynamic trajectory of the overall structure of the rat intestinal flora [57-59]. The LDA effect size (LEfSe) analysis was used to perform the quantitative analysis of the biomarkers for each group [60].

\subsection{Statistical Analysis}

The results were presented as means \pm the standard error of the mean (SEM). Statistical analysis was performed using SPSS, version 20 (IBM, Armonk, NY, USA). The differences between groups were statistically analyzed using ANOVA, followed by LSD multiple comparison tests and unpaired tests, and were considered statistically significant at a level of $p<0.05$.

\subsection{Accession Number}

The sequence information in this paper was submitted to the GenBank Sequence Read Archive database under accession number SRP020353.

\section{Conclusions}

In conclusion, our results show that wild rice treatment modulates the gut microbiota, promoting a decrease in the ratio of Firmicutes to Bacteroidetes, and increasing the relative abundance of Lactobacillus, while bringing the abundances of Prevotella, Alloprevotella, Anaerovibrio, and Staphylococcus back to normal levels, thereby providing beneficial effects against obesity, insulin resistance, liver steatosis, and low-grade inflammation to alter MAFLD in HFD-fed mice. 
Supplementary Materials: Supplementary materials can be found at http://www.mdpi.com/1422-0067/21/15/5375/ s1. Table S1: Composition of experimental diets; Table S2: List of primers used in this study; Table S3 Sequencing and data quality controls of each group; Table S4: The phylogeny and relaticve abundance of OTUs altered by wild rice, and the $\mathrm{p}$ value of LSD test evaluating the abundance difference of these OTUs between each group.

Author Contributions: X.-L.Y. and X.-D.H. conceived of and designed the study; N.Y., Y.-M.D., and H.L. performed the study, collected samples, and collected data; X.-L.Y. processed the microbiome sequencing data; X.-L.Y. and X.-D.H. analyzed the microbiome sequencing data; X.-D.H. and Z.-F.Z. analyzed the data; X.-L.Y. prepared the manuscript. All authors have read and agreed to the published version of the manuscript.

Funding: This work was supported by the Fundamental Research Funds for the Central Non-Profit Scientific Institution (grant no. 1610232019006 and 1610232020008), the Science Foundation for Young Scholars of Institute of Tobacco Research of Chinese Academy of Agricultural Sciences (grant number 2020A01), the Agricultural Science and Technology Innovation Program (grant no. ASTIP-TRIC05), and Key Projects of Wannan Tobacco Co., Ltd. (grant no. 20180023).

Conflicts of Interest: The authors have declared that no competing interests exist.

\section{References}

1. Eslam, M.; Newsome, P.N.; Sarin, S.K.; Anstee, Q.M.; Targher, G.; Romero-Gomez, M.; Zelber-Sagi, S.; Wong, V.W.-S.; Dufour, J.F.; Schattenberg, J.M.; et al. A new definition for metabolic dysfunction-associated fatty liver disease: An international expert consensus statement. J. Hepatol. 2020, 73, 202-209. [CrossRef] [PubMed]

2. Eslam, M.; Sanyal, A.J.; George, J. International Consensus Panel, MAFLD: A Consensus-Driven Proposed Nomenclature for Metabolic Associated Fatty Liver Disease. Gastroenterology 2020, 158, 1999-2014. [CrossRef] [PubMed]

3. Ye, Q.; Zou, B.; Yeo, Y.H.; Li, J.; Huang, D.Q.; Wu, Y.; Yang, H.; Liu, C.; Kam, L.Y.; Tan, X.X.E.; et al. Global prevalence, incidence, and outcomes of non-obese or lean non-alcoholic fatty liver disease: A systematic review and meta-analysis. Lancet Gastroenterol. Hepatol. 2020, 8, 739-752. [CrossRef]

4. Loomba, R.; Sanyal, A.J. The Global NAFLD Epidemic. Nat. Rev. Gastro. Hepat. 2013, 10, 686-690. [CrossRef]

5. Younossi, Z.M.; Blissett, D.; Blissett, R.; Henry, L.; Stepanova, M.; Younossi, Y.; Racila, A.; Hunt, S.; Beckerman, R. The economic and clinical burden of nonalcoholic fatty liver disease in the United States and Europe. Hepatology 2016, 64, 1577-1586. [CrossRef]

6. Spahis, S.; Alvarez, F.; Ahmed, N.; Dubois, J.; Jalbout, R.; Paganelli, M.; Grzywacz, K.; Delvin, E.; Peretti, N.; Levy, E. Non-alcoholic fatty liver disease severity and metabolic complications in obese children: Impact of omega-3 fatty acids. J. Nutr. Biochem. 2018, 58, 28-36. [CrossRef]

7. Tilg, H.; Moschen, A.R.; Roden, M. NAFLD and diabetes mellitus. Nat. Rev. Gastroenterol. Hepatol. 2017, 14, 32-42. [CrossRef]

8. Marcuccilli, M.; Chonchol, M. NAFLD and Chronic Kidney Disease. Int. J. Mol. Sci. 2016, 17, 562. [CrossRef]

9. Safari, Z.; Gérard, P. The links between the gut microbiome and non-alcoholic fatty liver disease (NAFLD). Cell. Mol. Life Sci. 2019, 76, 1541-1558. [CrossRef]

10. Esteve, E.; Ricart, W.; Fernándezreal, J.M. Gut microbiota interactions with obesity, insulin resistance and type 2 diabetes: Did gut microbiote co-evolve with insulin resistance? Curr. Opin. Clin. Nutr. 2011, 14, 483-490. [CrossRef]

11. Chang, C.J.; Lin, C.S.; Lu, C.C.; Martel, J.; Ko, Y.F.; Ojcius, D.M.; Tseng, S.F.; Wu, T.R.; Chen, Y.Y.M.; Young, J.D. Corrigendum: Ganoderma lucidum reduces obesity in mice by modulating the composition of the gut microbiota. Nat. Commun. 2017, 6, 7489. [CrossRef] [PubMed]

12. Méndez-Salazar, E.O.; Ortiz-López, M.G.; de los Ángeles Granados-Silvestre, M.; Palacios-Gonzalez, B.; Menjivar, M. Altered Gut Microbiota and Compositional Changes in Firmicutes and Proteobacteria in Mexican Undernourished and Obese Children. Front. Microbiol. 2018, 9, 2494. [CrossRef]

13. Chen, F.; Esmaili, S.; Rogers, G.B.; Bugianesi, E.; Petta, S.; Marchesini, G.; Bayoumi, A.; Metwally, M.; Azardaryany, M.K.; Coulter, S.; et al. Lean NAFLD: A Distinct Entity Shaped by Differential Metabolic Adaptation. Hepatology 2020, 71, 1213-1227. [CrossRef] [PubMed]

14. Milosevic, I.; Vujovic, A.; Barac, A.; Djelic, M.; Korac, M.; Radovanovic Spurnic, A.; Gmizic, I.; Stevanovic, O.; Djordjevic, V.; Lekic, N.; et al. Gut-Liver Axis, Gut Microbiota, and Its Modulation in the Management of Liver Diseases: A Review of the Literature. Int. J. Mol. Sci. 2019, 20, 395. [CrossRef] [PubMed] 
15. Leung, C.; Rivera, L.; Furness, J.B.; Angus, P.W. The role of the gut microbiota in NAFLD. Nat. Rev. Gastroenterol. Hepatol. 2016, 13, 412-425. [CrossRef] [PubMed]

16. Kim, T.T.; Parajuli, N.; Sung, M.M.; Bairwa, S.C.; Levasseur, J.; Soltys, C.M.; Wishart, D.S.; Madsen, K.; Schertzer, J.D.; Jrb, D. Fecal transplant from resveratrol-fed donors improves glycaemia and cardiovascular features of the metabolic syndrome in mice. Am. J. Physiol. Endocrinol. Metab. 2018, 315, 511-519. [CrossRef]

17. De Palma, G.; Lynch, M.D.; Lu, J.; Dang, V.T.; Deng, Y.; Jury, J.; Umeh, G.; Miranda, P.M.; Pastor, M.P.; Sidani, S. Transplantation of fecal microbiota from patients with irritable bowel syndrome alters gut function and behavior in recipient mice. Sci. Transl. Med. 2017, 9, eaaf6397. [CrossRef]

18. Zhang, H.; Cao, P.; Agellon, L.B.; Zhai, C.-K. Wild rice (Zizania latifolia (Griseb) Turcz) improves the serum lipid profile and antioxidant status of rats fed with a high fat/cholesterol diet. Brit. J. Nutr. 2009, 102, 1723-1727. [CrossRef]

19. Zhai, C.; Lu, C.; Zhang, X.; Sun, G.; Lorenz, K. Comparative study on nutritional value of Chinese and North American wild rice. Food Compos. Anal. 2001, 14, 371-382. [CrossRef]

20. Moghadasian, M.H.; Zhao, R.; Ghazawwi, N.; Le, K.; Apea-Bah, F.B.; Beta, T.; Shen, G.X. Inhibitory effects of North American wild rice on monocyte adhesion and inflammatory modulators in low-density lipoprotein receptor-knockout mice. J. Agric. Food Chem. 2017, 65, 9054-9060. [CrossRef]

21. Sumczynski, D.; Koubová, E.; Šenkárová, L.; Orsavová, J. Rice flakes produced from commercial wild rice: Chemical compositions, vitamin B compounds, mineral and trace element contents and their dietary intake evaluation. Food Chem. 2018, 264, 386-392. [CrossRef] [PubMed]

22. Anwar, F.; Zengin, G.; Alkharfy, K.; Marcu, M. Wild rice (Zizania sp.): A potential source of valuable ingredients for nutraceuticals and functional foods. Riv. Ital. Sostanze Gr. 2017, 565, 81-89.

23. Qiu, Y.; Liu, Q.; Beta, T. Antioxidant properties of commercial wild rice and analysis of soluble and insoluble phenolic acids. Food Chem. 2010, 121, 140-147. [CrossRef]

24. Qiu, Y.; Liu, Q.; Beta, T. Antioxidant activity of commercial wild rice and identification of flavonoid compounds in active fractions. J. Agric. Food Chem. 2009, 57, 7543-7551. [CrossRef]

25. Chu, M.J.; Liu, X.M.; Yan, N.; Wang, F.Z.; Du, Y.M.; Zhang, Z.F. Partial purification, identification, and quantitation of antioxidants from wild rice (Zizania latifolia). Molecules 2018, 23, 2782. [CrossRef]

26. Chu, M.J.; Du, Y.M.; Liu, X.M.; Yan, N.; Wang, F.Z.; Zhang, Z.F. Extraction of proanthocyanidins from Chinese wild rice (Zizania latifolia) and analyses of structural composition and potential bioactivities of different fractions. Molecules 2019, 24, 1681. [CrossRef]

27. Tao, W.; Zhang, Y.; Shen, X.; Cao, Y.; Shi, J.; Ye, X.; Chen, S. Rethinking the mechanism of the health benefits of proanthocyanidins: Absorption, metabolism, and interaction with gut microbiota. Compr. Rev. Food Sci. Food Saf. 2019, 18, 971-985. [CrossRef]

28. Ji, Y.; Chung, Y.M.; Park, S.; Jeong, D.; Kim, B.; Holzapfel, W.H. Dose dependent anti-obesity effect of three different Lactobacillus sakei strains using a diet induced obese murine model. PeerJ Prepr. 2018, 6, e26959v1.

29. Wickens, K.L.; Barthow, C.A.; Murphy, R.; Abels, P.R.; Maude, R.M.; Stone, P.R.; Mitchell, E.A.; Stanley, T.V.; Purdie, G.L.; Kang, J.M. Early pregnancy probiotic supplementation with Lactobacillus rhamnosus HN001 may reduce the prevalence of gestational diabetes mellitus: A randomised controlled trial. Brit. J. Nutr. 2017, 117, 804-813. [CrossRef]

30. Gao, C.; Major, A.; Rendon, D.; Lugo, M.; Jackson, V.; Shi, Z.; Mori-Akiyama, Y.; Versalovic, J. Histamine H2 receptor-mediated suppression of intestinal inflammation by probiotic Lactobacillus reuteri. MBio 2015, 6 . [CrossRef]

31. Han, S.-F.; Zhang, H.; Zhai, C.-K. Protective potentials of wild rice (Zizania latifolia (Griseb) Turcz) against obesity and lipotoxicity induced by a high-fat/cholesterol diet in rats. Food Chem. Toxicol. 2012, 50, 2263-2269. [CrossRef]

32. Han, S.; Zhang, H.; Qin, L.; Zhai, C. Effects of dietary carbohydrate replaced with wild rice (Zizania latifolia (Griseb) Turcz) on insulin resistance in rats fed with a high-fat/cholesterol diet. Nutrients 2013, 5, 552-564. [CrossRef] [PubMed]

33. Lee, S.-S.; Baek, Y.-S.; Eun, C.-S.; Yu, M.-H.; Baek, N.-I.; Chung, D.-k.; Bang, M.-H.; Yang, S.-A. Tricin derivatives as anti-inflammatory and anti-allergic constituents from the aerial part of Zizania latifolia. Biosci. Biotech. Bioch. 2015, 79, 700-706. [CrossRef] [PubMed] 
34. Sonnenburg, E.D.; Smits, S.A.; Tikhonov, M.; Higginbottom, S.K.; Wingreen, N.S.; Sonnenburg, J.L. Diet-induced extinctions in the gut microbiota compound over generations. Nature 2016, 529, 212. [CrossRef] [PubMed]

35. Etxeberria, U.; Arias, N.; Boqué, N.; Macarulla, M.; Portillo, M.; Martínez, J.; Milagro, F. Reshaping faecal gut microbiota composition by the intake of trans-resveratrol and quercetin in high-fat sucrose diet-fed rats. J. Nutr. Biochem. 2015, 26, 651-660. [CrossRef]

36. Yang, J.; Lee, Y.; Kim, Y.; Lee, S.; Ryu, S.; Fukuda, S.; Hase, K.; Yang, C.; Lim, H.; Kim, M. Gut commensal Bacteroides acidifaciens prevents obesity and improves insulin sensitivity in mice. Mucosal Immunol. 2017, 10, 104. [CrossRef] [PubMed]

37. Flint, H.J. The impact of nutrition on the human microbiome. Nutr. Rev. 2012, 70, S10-S13. [CrossRef]

38. Holscher, H.D. Dietary fiber and prebiotics and the gastrointestinal microbiota. Gut Microbes 2017, 8, $172-184$. [CrossRef]

39. Yang, X.; Kwame, O.D.; Huang, Y.; He, C.; Yang, H.; He, S.; Li, J.; Li, J.; Berthold, H.; Yin, Y. Resistant Starch Regulates Gut Microbiota: Structure, Biochemistry and Cell Signalling. Cell. Physiol. Biochem. 2017, 42, 306-318. [CrossRef]

40. Błażejewski, A.J.; Thiemann, S.; Schenk, A.; Pils, M.C.; Gálvez, E.J.; Roy, U.; Heise, U.; de Zoete, M.R.; Flavell, R.A.; Strowig, T. Microbiota normalization reveals that canonical caspase-1 activation exacerbates chemically induced intestinal inflammation. Cell Rep. 2017, 19, 2319-2330. [CrossRef]

41. Storelli, G.; Strigini, M.; Grenier, T.; Bozonnet, L.; Schwarzer, M.; Daniel, C.; Matos, R.; Leulier, F. Drosophila perpetuates nutritional mutualism by promoting the fitness of its intestinal symbiont Lactobacillus plantarum. Cell Metab. 2018, 27, 362-377. [CrossRef] [PubMed]

42. Khare, P.; Jagtap, S.; Jain, Y.; Baboota, R.K.; Mangal, P.; Boparai, R.K.; Bhutani, K.K.; Sharma, S.S.; Premkumar, L.S.; Kondepudi, K.K.; et al. Cinnamaldehyde supplementation prevents fasting-induced hyperphagia, lipid accumulation, and inflammation in high-fat diet-fed mice. Biofactors 2016, 42, 201-211.

43. Nihei, N.; Okamoto, H.; Furune, T.; Ikuta, N.; Sasaki, K.; Rimbach, G.; Yoshikawa, Y.; Terao, K. Dietary $\alpha$-cyclodextrin modifies gut microbiota and reduces fat accumulation in high-fat-diet-fed obese mice. Biofactors 2018, 44, 336-347. [CrossRef] [PubMed]

44. Wu, C.; Tian, Y.; Yu, J.; Zhang, R.; Zhang, X.; Guo, P. The pandanus tectorius fruit extract (PTF) modulates the gut microbiota and exerts anti-hyperlipidaemic effects. Phytomedicine 2019, 58, 152863. [CrossRef] [PubMed]

45. Singh, D.P.; Singh, J.; Boparai, R.K.; Zhu, J.; Mantri, S.; Khare, P.; Khardori, R.; Kondepudi, K.K.; Chopra, K.; Bishnoi, M. Isomalto-oligosaccharides, a prebiotic, functionally augment green tea effects against high fat diet-induced metabolic alterations via preventing gut dysbacteriosis in mice. Pharm. Res. 2017, 123, 103-113. [CrossRef]

46. Hjorth, M.; Roager, H.M.; Larsen, T.; Poulsen, S.; Licht, T.R.; Bahl, M.I.; Zohar, Y.; Astrup, A. Pre-treatment microbial Prevotella-to-Bacteroides ratio, determines body fat loss success during a 6-month randomized controlled diet intervention. Int. J. Obes. 2018, 42, 580. [CrossRef]

47. Rios-Covian, D.; Salazar, N.; Gueimonde, M.; de los Reyes-Gavilan, C.G. Shaping the metabolism of intestinal Bacteroides population through diet to improve human health. Front. Microbiol. 2017, 8, 376. [CrossRef]

48. Larsen, J.M. The immune response to Prevotella bacteria in chronic inflammatory disease. Immunology 2017, 151, 363-374. [CrossRef]

49. Domingueti, C.P.; Dusse, L.M.S.A.; das Graças Carvalho, M.; de Sousa, L.P.; Gomes, K.B.; Fernandes, A.P. Diabetes mellitus: The linkage between oxidative stress, inflammation, hypercoagulability and vascular complications. J. Diabetes Complicat. 2016, 30, 738-745. [CrossRef]

50. Cooke, A.A.; Connaughton, R.M.; Lyons, C.L.; McMorrow, A.M.; Roche, H.M. Fatty acids and chronic low grade inflammation associated with obesity and the metabolic syndrome. Eur. J. Pharmacol. 2016, 785, 207-214. [CrossRef]

51. Kilkenny, C.; Browne, W.J.; Cuthill, I.C.; Emerson, M.; Altman, D.G. Improving bioscience research reporting: The ARRIVE guidelines for reporting animal research. PLoS Biol. 2010, 8, e1000412. [CrossRef] [PubMed]

52. Maleszewski, J.J.; Orellana, V.M.; Hodge, D.O.; Kuhl, U.; Schultheiss, H.-P.; Cooper, L.T. Long-term risk of recurrence, morbidity and mortality in giant cell myocarditis. Am. J. Cardiol. 2015, 115, 1733-1738. [CrossRef] [PubMed] 
53. Yuan, X.-L.; Zhang, P.; Liu, X.-M.; Du, Y.-M.; Hou, X.-D.; Cheng, S.; Zhang, Z.-F. Cytological assessments and transcriptome profiling demonstrate that evodiamine inhibits growth and induces apoptosis in a renal carcinoma cell line. Sci. Rep. 2017, 7, 12572. [CrossRef] [PubMed]

54. Xu, P.; Zhang, Y.; Liu, Y.; Yuan, Q.; Song, L.; Liu, M.; Liu, Z.; Yang, Y.; Li, J.; Li, D. Fibroblast growth factor 21 attenuates hepatic fibrogenesis through TGF- $\beta / \mathrm{smad} 2 / 3$ and NF- $\mathrm{B}$ signaling pathways. Toxicol. Appl. Pharm. 2016, 290, 43-53. [CrossRef]

55. Magoč, T.; Salzberg, S.L. FLASH: Fast length adjustment of short reads to improve genome assemblies. Bioinformatics 2011, 27, 2957-2963. [CrossRef]

56. Edgar, R.C.; Haas, B.J.; Clemente, J.C.; Quince, C.; Knight, R. UCHIME improves sensitivity and speed of chimera detection. Bioinformatics 2011, 27, 2194-2200. [CrossRef]

57. Lozupone, C.A.; Hamady, M.; Kelley, S.T.; Knight, R. Quantitative and qualitative $\beta$ diversity measures lead to different insights into factors that structure microbial communities. Appl. Environ. Microbiol. 2007, 73, 1576-1585. [CrossRef]

58. RC Team. R: A Language and Environment for Statistical Computing; MSOR Connections; RC Team: Vienna, Austria, 2014; Volume 1.

59. Mahnert, A.; Moissl-Eichinger, C.; Berg, G. Microbiome interplay: Plants alter microbial abundance and diversity within the built environment. Front. Microbiol. 2015, 6, 887. [CrossRef]

60. Segata, N.; Izard, J.; Waldron, L.; Gevers, D.; Miropolsky, L.; Garrett, W.S.; Huttenhower, C. Metagenomic biomarker discovery and explanation. Genome Biol. 2011, 12, 1-18. [CrossRef]

(C) 2020 by the authors. Licensee MDPI, Basel, Switzerland. This article is an open access article distributed under the terms and conditions of the Creative Commons Attribution (CC BY) license (http://creativecommons.org/licenses/by/4.0/). 